\title{
Poiseuille flow in curved spaces
}

\author{
J.-D. Debus, ${ }^{1, *}$ M. Mendoza,${ }^{1, \dagger}$ S. Succi, ${ }^{2}, \ddagger$ and H. J. Herrmann ${ }^{1, \S}$ \\ ${ }^{1}$ ETH Zürich, Computational Physics for Engineering Materials, \\ Institute for Building Materials, Wolfgang-Pauli-Strasse 27, HIT, CH-8093 Zürich, Switzerland \\ ${ }^{2}$ Instituto per le Applicazioni del Calcolo C.N.R., Via dei Taurini, 19 00185, Rome, Italy
}

\begin{abstract}
We investigate Poiseuille channel flow through intrinsically curved media, equipped with localized metric perturbations. To this end, we study the flux of a fluid driven through the curved channel in dependence of the spatial deformation, characterized by the parameters of the metric perturbations (amplitude, range and density). We find that the flux depends only on a specific combination of parameters, which we identify as the average metric perturbation, and derive a universal flux law for the Poiseuille flow. For the purpose of this study, we have improved and validated our recently developed lattice Boltzmann model in curved space by considerably reducing discrete lattice effects.
\end{abstract}

PACS numbers: 47.11.-j, 02.40.-k

\section{INTRODUCTION}

In many physical systems in Nature, the motion of particles is restricted to a given manifold (e.g., to a twodimensional surface). For example, soap bubbles are commonly described as a two-dimensional fluid moving on a spherical surface, since the perpendicular radial dimension is negligibly small [1]. The same concept applies to curved two-dimensional interfaces, e.g., molecular films around an emulsion or aerosol droplet, soap films or foam bubbles, the Earth's atmosphere or the photosphere of the sun [2-6]. Because of their experimental accessibility, soap films are of particular interest for the study of 2D surface flow [7], as they can be fabricated on large scales [8] and provide the possibility to study custom-made curvature effects on the flow experimentally. Another important field of application is the dynamics of lipid bilayers in microbiology [9]. These lipid bilayers are of particular importance since they constitute the envelope of most of the cell components. In particular, lipid bilayer membranes are known to enter a fluid phase above a transition temperature [9] and can thus be described as a viscous two-dimensional fluid moving along the curved membrane [10-12], as has been confirmed experimentally [13-15]. Besides that, electron transport on $2 \mathrm{D}$ graphene sheets is known to follow the Navier-Stokes equation in the hydrodynamic regime [16, 17]. Since graphene sheets can form ripples [18], a natural extension to describe electron flow on curved graphene sheets is the Navier-Stokes equation in curved space. Thinking of cosmological scales, space-time itself can be seen as a four-dimensional, intrinsically curved medium whose curvature is caused by the matter it contains according to Einstein's field equations [19]. As a consequence, flow of cosmic dust is deflected in the gravitational and spacecurving field of the surrounding stars.

\footnotetext{
* debusj@ethz.ch

$\dagger$ mmendoza@ethz.ch

$\ddagger$ succi@iac.cnr.it

$\S$ hjherrmann@ethz.ch
}

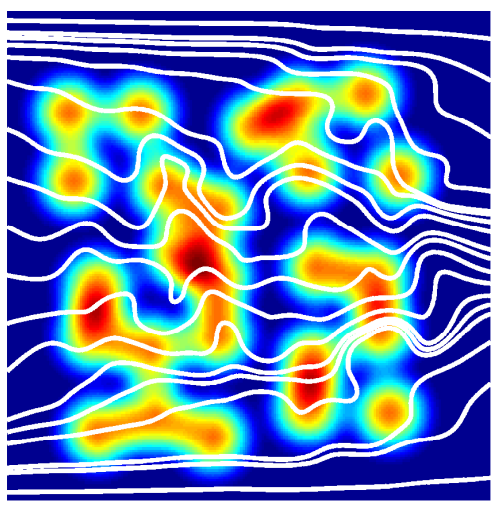

Figure 1. Velocity streamlines of a fluid driven through a curved medium with randomly distributed metric perturbations. The colors represent the strength of the perturbation $\delta g$ added to the diagonal components of the metric tensor, ranging from flat space (blue) to high perturbation (red).

Recently, we have found that local sources of curvature induce dissipation within a viscous fluid [20]. In this paper, we study a different setup, namely Poiseuillelike channel flow through an intrinsically curved space, where the dissipation exerted by the walls dominates over the dissipation induced by the metric perturbations, and therefore, the latter can be neglected. Starting from flat space, we introduce local sources of curvature by adding localized perturbations $\delta g$ to the Riemann metric $g$. Note that these perturbations do not mimic solid obstacles or boundary walls, since the curved regions of space are still permeable to the flow and thus cannot mimic noslip boundary conditions. Instead, the metric perturbations lead to an intrinsic curvature of space. The fluid is driven through the curved channel according to the Navier-Stokes equations in curved space, as illustrated in Fig. 1. For different configurations of metric perturbations, we measure the flux of the Poiseuille-like flow in the stationary state and establish a connection between the flux and the curvature parameters. To this end, we consider a two-dimensional channel with regularly and ran- 
domly arranged metric perturbations (see Figs. 2 and 6 for a sketch), which are characterized by their amplitude $a_{0}$, range $r_{0}$, and number density $n$. We find that the flux depends only on the specific combination of parameters, which we identify as the average metric perturbation $\langle\delta g\rangle \sim a_{0} r_{0}^{2} n$. Plotting the flux as function of $\langle\delta g\rangle$, we observe that all the data points collapse onto a single curve, which we describe by an empirical flux law. Secondly, we investigate flow through a three-dimensional curved channel, in order to clarify the influence of the spatial dimension. In three dimensions, we find an analogous flux law to the two-dimensional case.

For the simulations, we use the lattice Boltzmann (LB) method, which describes the motion of a fluid from the perspective of kinetic theory (for a review of the standard LB method see Ref. [21-23]). Recently, the LB method has been extended to general Riemannian manifolds [2426], allowing us to simulate fluid flow in nearly arbitrary, smooth geometries. Here, we have further improved the method in Ref. [26] by improving the treatment of discrete lattice effects, which originate from the forcing term of the LB equation and which lead to spurious source terms in the Navier-Stokes equations (see also Ref. [27]). By significantly reducing the discrete lattice effects, we have been able to improve the accuracy of our method considerably.

The paper is organized as follows: In Sec. II we review the lattice Boltzmann method in curved space and present an improved model in which spurious discrete lattice effects are canceled at order $\Delta t$. Next, in Sec. III, we study channel flow through different types of curved media, starting from two-dimensional media with regularly and randomly arranged metric perturbations and ending with three-dimensional media. We compare the results to a previous study in Ref. [24]. Finally, we present a validation of the model by showing the improvement of the method with respect to discrete lattice effects and by confirming that our results are not biased by finite resolution effects.

\section{LATTICE BOLTZMANN METHOD}

\section{A. Review: Lattice Boltzmann in curved space}

In this section, we present a short summary of the method used to simulate Poiseuille flow on Riemann manifolds, equipped with a stationary Riemann metric $g$. More details and validations of the method can be found in previous publications [24-26]. We will use the following notation for partial derivatives throughout the whole paper:

$$
\partial_{t}:=\frac{\partial}{\partial t}, \quad \partial_{i}:=\frac{\partial}{\partial x^{i}}
$$

Furthermore, we use the Einstein sum convention, i.e. repeated indices are summed over. At first, we specify

\begin{tabular}{|c|c|c|}
\hline $\bar{\lambda}$ & $\vec{c}_{\lambda}$ & $w_{\lambda}$ \\
\hline 1 & $(0,0,0)$ & $\frac{2}{2025}(5045-1507 \sqrt{10})$ \\
\hline 2,3 & $( \pm 1,0,0)$ & \multirow{3}{*}{$\frac{37}{5 \sqrt{10}}-\frac{91}{40}$} \\
\hline 4,5 & $(0, \pm 1,0)$ & \\
\hline 6,7 & $(0,0, \pm 1)$ & \\
\hline 8-11 & $( \pm 1, \pm 1,0)$ & \multirow{3}{*}{$\frac{1}{50}(55-17 \sqrt{10})$} \\
\hline $12-15$ & $( \pm 1,0, \pm 1)$ & \\
\hline $16-19$ & $(0, \pm 1, \pm 1)$ & \\
\hline $20-27$ & $( \pm 1, \pm 1, \pm 1)$ & $\frac{1}{1600}(233 \sqrt{10}-730)$ \\
\hline 28,29 & $( \pm 3,0,0)$ & \multirow{3}{*}{$\frac{1}{16200}(295-92 \sqrt{10})$} \\
\hline 30,31 & $(0, \pm 3,0)$ & \\
\hline 32,33 & $(0,0, \pm 3)$ & \\
\hline $34-41$ & $( \pm 3, \pm 3, \pm 3)$ & $\frac{1}{129600}(130-41 \sqrt{10})$ \\
\hline
\end{tabular}

Table I. Discrete velocity vectors $c_{\lambda}$ of the D3Q41 lattice and the corresponding weights $w_{\lambda}$ for the Hermite quadrature.

a $D$-dimensional coordinate system by choosing local coordinates $\left(x^{1}, x^{2}, \ldots, x^{D}\right)$ with the corresponding standard vector basis $\left(\vec{e}_{1}, \vec{e}_{2}, \ldots, \vec{e}_{D}\right)=\left(\frac{\partial}{\partial x^{1}}, \frac{\partial}{\partial x^{2}}, \ldots, \frac{\partial}{\partial x^{D}}\right)$. In the following, all vectorial and tensorial quantities will be expressed in this basis, e.g. the components of the Riemann metric tensor $g$ are given by $g_{i j}=g\left(\vec{e}_{i}, \vec{e}_{j}\right)$.

The basic equation in our model is the lattice Boltzmann equation in curved space,

$f_{\lambda}\left(\vec{r}+\vec{c}_{\lambda} \Delta t, t+\Delta t\right)-f_{\lambda}(\vec{r}, t)=-\frac{1}{\tau}\left(f_{\lambda}-f_{\lambda}^{\mathrm{eq}}\right)+\Delta t \mathcal{F}_{\lambda}$,

which describes the evolution of a distribution function $f_{\lambda}(\vec{r}, t)$ on a lattice. Here $\vec{r}=\left(x^{1}, x^{2}, \ldots, x^{D}\right)$ denotes the lattice position and $\lambda$ labels the discrete lattice velocities corresponding to the velocity vectors $\vec{c}_{\lambda}$. We use the Bhatnagar-Gross-Krook (BGK) approximation [28] for the description of the particle collisions, meaning that all collisions are characterized by a single-relaxation time $\tau$. The Maxwell-Boltzmann equilibrium distribution is denoted by $f_{\lambda}^{\mathrm{eq}}$, and $\mathcal{F}_{\lambda}$ represents a forcing term, which accounts for the inertial forces in a curved space. Since we perform simulations in both two- and three-dimensions, we choose the D3Q41 lattice [29]. By using this special lattice, the moments of the distribution function are exactly preserved up to third order due to the properties of Gauss-Hermite quadrature (see Appendix A 1). The lattice contains 41 discrete velocity vectors $\left\{\vec{c}_{\lambda}\right\}$ (see Table I) and the speed of sound is given by $c_{s}^{2}=1-\sqrt{2 / 5}$. The macroscopic fluid density $\rho$ and fluid velocity $\vec{u}$ are obtained from the moments of the distribution function,

$$
\sum_{\lambda=1}^{41} \sqrt{g} f_{\lambda}=\rho \quad, \quad \sum_{\lambda=1}^{41} \sqrt{g} \vec{c}_{\lambda} f_{\lambda}=\rho \vec{u},
$$

where $\sqrt{g}$ is the square root of the determinant of the metric, originating from the integration measure on manifolds, $d V=\sqrt{g} d x^{1} \cdots d x^{D}$. The density $\rho$ and velocity $u^{i}$ fulfill the hydrodynamic conservation equations, which 
on a manifold read

$$
\partial_{t} \rho+\nabla_{i}\left(\rho u^{i}\right)=0, \quad \partial_{t}\left(\rho u^{i}\right)+\nabla_{j} T^{i j}=0,
$$

(covariant Navier-Stokes equations), where $\nabla$ denotes the covariant derivative (Levi-Civita connection) and $T^{i j}$ is the energy-stress tensor. In the incompressible limit, the energy-stress tensor is given by

$$
\begin{aligned}
& T^{i j}=\Pi^{\mathrm{eq}, i j}-\sigma^{i j} \\
& =\left(P g^{i j}+\rho u^{i} u^{j}\right)-\nu\left(\nabla^{j}\left(\rho u^{i}\right)+\nabla^{i}\left(\rho u^{j}\right)+g^{i j} \nabla_{k}\left(\rho u^{k}\right)\right),
\end{aligned}
$$

where in the first line $\Pi^{\mathrm{eq}, i j}$ is the free momentum-flux tensor and $\sigma^{i j}$ denotes the viscous stress tensor. In the second line, $P=\rho \theta$ denotes the hydrostatic pressure, $\theta$ the normalized temperature, $\nu$ the kinematic viscosity, and $g^{i j}$ the components of the inverse metric tensor. (Note that the method is capable to handle very small but non-vanishing compressibilities, thus we keep terms like $\nabla_{k}\left(\rho u^{k}\right)$ in the equations although they are negligible.) We further work in the isothermal limit $\theta=1$.

The explicit discrete form of the equilibrium distribution is given by an expansion in tensor Hermite polynomials,

$$
\begin{aligned}
f_{\lambda}^{\mathrm{eq}} & =\frac{w_{\lambda}}{\sqrt{g}}\left(a_{(0)}^{\mathrm{eq}}+\frac{1}{c_{s}^{2}} a_{(1)}^{\mathrm{eq}, i} c_{\lambda}^{i}+\frac{1}{2 ! c_{s}^{4}} a_{(2)}^{\mathrm{eq}, i j}\left(c_{\lambda}^{i} c_{\lambda}^{j}-c_{s}^{2} \delta^{i j}\right)\right. \\
& \left.+\frac{1}{3 ! c_{s}^{6}} a_{(3)}^{\mathrm{eq}, i j k}\left(c_{\lambda}^{i} c_{\lambda}^{j} c_{\lambda}^{k}-c_{s}^{2}\left(\delta^{i j} c_{\lambda}^{k}+\delta^{j k} c_{\lambda}^{i}+\delta^{k i} c_{\lambda}^{j}\right)\right)\right)
\end{aligned}
$$

where the expansion coefficients read as follows:

$$
\begin{aligned}
& a_{(0)}^{\mathrm{eq}}=\rho, \quad a_{(1)}^{\mathrm{eq}, i}=\rho u^{i}, \quad a_{(2)}^{\mathrm{eq}, i j}=\rho c_{s}^{2} \Delta^{i j}+\rho u^{i} u^{j}, \\
& a_{(3)}^{\mathrm{eq}, i j k}=\rho c_{s}^{2}\left(\Delta^{i j} u^{k}+\Delta^{j k} u^{i}+\Delta^{k i} u^{j}\right)+\rho u^{i} u^{j} u^{k} .
\end{aligned}
$$

Here, $w_{\lambda}$ are the lattice weights (see Table I) and $\Delta^{i j}:=$ $g^{i j}-\delta^{i j}$ is a measure of the deviation from flat space.

The forcing term $\mathcal{F}_{\lambda}$ is also expanded into Hermite polynomials,

$$
\begin{aligned}
\mathcal{F}_{\lambda} & =\frac{w_{\lambda}}{\sqrt{g}}\left(\frac{1}{c_{s}^{2}} a_{(0)} F_{\lambda}^{i} c_{\lambda}^{i}+\frac{1}{c_{s}^{4}} a_{(1)}^{i} F_{\lambda}^{j}\left(c_{\lambda}^{i} c_{\lambda}^{j}-c_{s}^{2} \delta^{i j}\right)\right. \\
& \left.+\frac{1}{2 c_{s}^{6}} a_{(2)}^{i j} F_{\lambda}^{k}\left(c_{\lambda}^{i} c_{\lambda}^{j} c_{\lambda}^{k}-c_{s}^{2}\left(\delta^{i j} c_{\lambda}^{k}+\delta^{j k} c_{\lambda}^{i}+\delta^{k i} c_{\lambda}^{j}\right)\right)\right)
\end{aligned}
$$

where $F_{\lambda}^{i}=-\Gamma_{j k}^{i} c_{\lambda}^{j} c_{\lambda}^{k}$ represents the inertial forces driving the fluid along the geodesics of the curved space, and $\Gamma_{j k}^{i}$ are the Christoffel symbols. The expansion coefficients of the forcing term are given by

$$
a_{(0)}=a_{(0)}^{\mathrm{eq}}, \quad a_{(1)}^{i}=a_{(1)}^{\mathrm{eq}, i}, \quad a_{(2)}^{i j}=a_{(2)}^{\mathrm{eq}, i j}-\sigma^{i j},
$$

where $\sigma^{i j}=-\left(1-\frac{1}{2 \tau}\right) \sum_{\lambda} \sqrt{g} c_{\lambda}^{i} c_{\lambda}^{j}\left(f_{\lambda}-f_{\lambda}^{\text {eq }}\right)$ denotes the viscous stress tensor on the lattice. External forces (needed to drive the fluid through the medium) are added to the lattice Boltzmann equation as described in Sec. II B.
From the metric tensor $g_{i j}$, the Christoffel symbols are calculated by

$$
\Gamma_{j k}^{i}=\frac{1}{2} g^{i m}\left(\partial_{k} g_{j m}+\partial_{j} g_{k m}-\partial_{m} g_{j k}\right),
$$

where the spatial derivatives of the metric can be computed very accurately by using isotropic lattice differentiation operators developed in Ref. [30], for example

$$
\partial_{k} g_{j m}(\vec{r})=\frac{1}{c_{s}^{2} \Delta t} \sum_{\lambda=1}^{41} w_{\lambda} c_{\lambda}^{k} g_{j m}\left(\vec{r}+\vec{c}_{\lambda} \Delta t\right)
$$

Having all ingredients at hand for the LB Eq. (1), the LB algorithm can be applied as usual: After assigning initial conditions to the macroscopic quantities $\rho$ and $\vec{u}$, the distribution function $f$ is successively updated time step by time step according to the LB equation.

\section{B. Cancelation of discrete lattice effects}

As already known from the lattice Boltzmann methods in flat Cartesian space [27], discrete lattice effects arise when a force is introduced into the lattice Boltzmann equation. These discrete lattice effects manifest themselves as spurious additional terms of order $\Delta t$ in the Navier-Stokes equations:

$$
\begin{aligned}
\partial_{t} \rho+\nabla_{i}\left(\rho u^{i}\right) & =-\frac{\Delta t}{2}\left(\partial_{t} A+\left(\partial_{i}-\Gamma_{i j}^{j}\right) B^{i}\right), \\
\partial_{t}\left(\rho u^{i}\right)+\nabla_{j} T^{i j} & =-\frac{\Delta t}{2} \partial_{t} B^{i},
\end{aligned}
$$

where $A:=\sum_{\lambda} \sqrt{g} \mathcal{F}_{\lambda}$ and $B^{i}:=\sum_{\lambda} \sqrt{g} \mathcal{F}_{\lambda} c_{\lambda}^{i}$ are the moments of the forcing term $\mathcal{F}_{\lambda}$. As can be seen, the terms on the right-hand side of the equations act as spurious source terms and should thus be canceled. If the external force only depends on space and time (but not on the fluid velocity), this can be done through a simple redefinition of the fluid velocity (see Ref. [27]). In our case, however, the inertial forces, $F_{\lambda}^{i}=-\Gamma_{j k}^{i} c_{\lambda}^{j} c_{\lambda}^{k}$, also depend on the microscopic fluid velocities $c_{\lambda}^{i}$, which affects the moments of the forcing term crucially. Moreover, our forcing term contributes not only to the momentum equation but also to the continuity equation, thus violating one of the necessary conditions on the forcing term considered in Ref. [27]. For all these reasons, the spurious discrete lattice effects in our model cannot be canceled as straightforwardly as in the simple case considered in Ref. [27]. However, it is still possible to eliminate spurious terms of order $\Delta t$ by correcting not only the velocity $\vec{u}$ but also the density $\rho$ at each time step:

$$
\begin{aligned}
\rho & \longrightarrow \hat{\rho}=\rho+\Delta t R(\hat{\rho}, \hat{u}), \\
\rho u^{i} \quad \longrightarrow \quad \hat{\rho} \hat{u}^{i} & =\rho u^{i}+\Delta t U^{i}(\hat{\rho}, \hat{u}),
\end{aligned}
$$


where $R(\hat{\rho}, \hat{u})$ and $U^{i}(\hat{\rho}, \hat{u})$ are correction terms, given by

$$
\begin{aligned}
R(\hat{\rho}, \hat{u}) & =-\frac{1}{2}\left(\Gamma_{i j}^{i} \hat{\rho} \hat{u}^{j}+\Gamma_{j i}^{i} \hat{\rho} \hat{u}^{j}\right), \\
U^{i}(\hat{\rho}, \hat{u}) & =-\frac{1}{2}\left(\Gamma_{j k}^{i} \Pi^{\mathrm{eq}, j k}+\Gamma_{j k}^{j} \Pi^{\mathrm{eq}, k i}+\Gamma_{k j}^{j} \Pi^{\mathrm{eq}, k i}\right),
\end{aligned}
$$

where $\Pi^{\text {eq, } i j}=\hat{\rho} c_{s}^{2} g^{i j}+\hat{\rho} \hat{u}^{i} \hat{u}^{j}$ denotes the free momentum-flux tensor. The correction terms $R$ and $U^{i}$ correspond to the moments of the forcing term and account for inertial effects on the fluid velocity and on the energy-stress tensor, which originate from the covariant derivatives in the Navier-Stokes Eqs. (2). Equations (7) and (8) define a system of coupled quadratic equations in $\hat{u}$, which can be solved numerically for $\hat{\rho}$ and $\hat{u}$ (e.g. using Newton's algorithm, which converges to the solution rapidly after a few iterations; see Appendix A 6). Additionally, the forcing term Eq. (4) is rescaled as follows:

$$
\mathcal{F}_{\lambda} \quad \longrightarrow \quad\left(1-\frac{1}{2 \tau}\right) \mathcal{F}_{\lambda}
$$

The equilibrium distribution as well as the forcing term are evaluated with the corrected density and velocity at each time step, $f^{\text {eq }}=f^{\text {eq }}(\hat{\rho}, \hat{u}), \mathcal{F}=\mathcal{F}(\hat{\rho}, \hat{u})$. An additional external force $\vec{F}^{\text {ext }}$ can be added straightforwardly to the lattice Boltzmann equation, replacing $F_{\lambda}^{i}$ by $\left(F_{\lambda}^{i}+F_{\text {ext }}^{i}\right)$ in the forcing term Eq. (4), which also results in an additional term in the correction function for the macroscopic velocity:

$$
U^{i}(\hat{\rho}, \hat{u}) \quad \longrightarrow \quad U^{i}(\hat{\rho}, \hat{u})+\frac{1}{2} \hat{\rho} F_{\text {ext }}^{i}
$$

As can be shown rigorously by a Chapman-Enskog expansion (see Appendix A for the details), these corrections lead to the correct macroscopic Eqs. (2), where spurious terms of order $\Delta t$ are canceled. In Sec. V A, we show that by using the corrected density Eq. (7) and velocity Eq. (8) as well as the modified forcing term Eq. (9), the method is improved considerably. In the following, we will drop the ${ }^{\wedge}$ symbol for the corrected density and velocity for simplicity.

\section{POISEUILLE FLOW IN CURVED SPACE}

In this section, we study the Poiseuille-like channel flow in curved space by investigating flow through different types of curved space. To this end, we disturb the flat space by adding local perturbations to the metric tensor, resulting in a physical curvature of space. This has to be distinguished from a simple coordinate transformation into curvilinear coordinates, which would not introduce spatial curvature. In the following, we will work in Cartesian-like coordinates $\left(x^{1}, x^{2}, x^{3}\right)=(x, y, z)$, such that the metric tensor of the flat space reads $g_{0}=\mathbb{1}$. The perturbed metric tensor with $N$ perturbations (labeled by an index $i$ ) is then given by

$$
g=(1+\delta g) \cdot \mathbb{1}=\left(1+\sum_{i=1}^{N} \delta g_{i}\right) \cdot \mathbb{1},
$$

where $\delta g_{i}$ denotes the contribution of the $i$ th perturbation. The individual metric perturbations are chosen to be of the shape

$$
\delta g_{i}= \begin{cases}-a_{0} \cos ^{2}\left(\pi \frac{r_{i}}{r_{0}}\right), & r_{i} \leq \frac{r_{0}}{2} \\ 0, & r_{i}>\frac{r_{0}}{2}\end{cases}
$$

where $r_{i}=\left\|\vec{r}-\vec{r}_{i}\right\|$ denotes the distance to the center $\vec{r}_{i}$ of the $i$-th perturbation. The parameters $a_{0}$ and $r_{0}$ characterize the amplitude and the range of the perturbation, respectively. We further define the density $n$ of the perturbations by $n=N / V_{0}$, where $V_{0}$ denotes the volume of the flat background medium. Since the individual perturbations possess a rotational symmetry, we will call this type "radial perturbations." In the simulations, we model a channel with periodic boundary conditions in the streamwise direction (at $x=0, x=L_{x}$ ) and fixed walls at the perpendicular boundaries (at $y=0, y=L_{y}$ and $z=$ $0, z=L_{z}$ ). At the walls, we impose zero-velocity boundary conditions on the fluid, which enters the algorithm through the equilibrium distribution $f^{\text {eq }}$ being evaluated with $u=0$ at the wall nodes. The fluid is driven through the medium by a constant external force corresponding to a pressure drop of $\nabla P=10^{-5}$ in $x$ direction. For all the two-dimensional simulations, we use a $D 3 Q 41$ lattice of size $L_{x} \times L_{y} \times L_{z}=256 \times 256 \times 1$, and set the discretization step to $\Delta t=\Delta x^{i}=128 / L_{x}$. For the viscosity of the fluid, we choose $\nu=c_{s}^{2}\left(\tau-\frac{1}{2}\right) \Delta t=c_{s}^{2} / 4$, which fixes the relaxation parameter $\tau$ correspondingly. The threedimensional simulations, on the other hand, are performed on a lattice of size $L_{x} \times L_{y} \times L_{z}=64 \times 64 \times 64$ with $\Delta t=\Delta x=\Delta y=\Delta z=1$ and $\nu=c_{s}^{2}\left(\tau-\frac{1}{2}\right) \Delta t=c_{s}^{2} / 2$.

After the fluid has reached the stationary state, we measure the conserved flux of the flow, which in curved space is given by

$$
\Phi=\int_{S} g\left(\rho \vec{u}, \vec{n}_{x}\right) d S=\int_{S} \rho u^{x} \sqrt{g} d y d z,
$$

where $\vec{n}_{x}=\vec{e}_{x} / \sqrt{g_{x x}}$ denotes the unit vector in $x$ direction and the integral runs over a channel cross-section with area element $d S=\sqrt{g_{y y} g_{z z}} d y d z$ and total area $S=\int_{S} d S$. Since we want to study the transport properties independently of the specific size of the medium, we consider the spatial average of the flux, given by

$$
\langle\Phi\rangle=\frac{1}{S} \int_{S} g\left(\rho \vec{u}, \vec{n}_{x}\right) d S=\frac{\Phi}{S} .
$$

In particular, we are interested in the relative deviation of the flux from the corresponding flux of the standard Poiseuille flow in flat space, $\Phi_{0}$, which is described by

$$
\Delta \Phi:=\frac{\left\langle\Phi_{0}\right\rangle-\langle\Phi\rangle}{\left\langle\Phi_{0}\right\rangle} .
$$




\section{A. 2D curved media}

For simplicity we start with a two-dimensional periodic arrangement of metric perturbations, where the positions $\vec{r}_{n}$ of the perturbations are confined to a regular square lattice,

$$
\vec{r}_{n}=\vec{r}_{n_{x}, n_{y}}=\left(n_{x}, n_{y}\right) \cdot \lambda, \quad n_{x}, n_{y} \in \mathbb{Z},
$$

where $\lambda=\sqrt{V_{0} / N}$ denotes the characteristic distance between the perturbations and $V_{0}=L_{x} L_{y} \Delta x \Delta y$ is the volume of the flat background space (see Fig. 2 for a sketch). Because of the periodicity of the metric per-

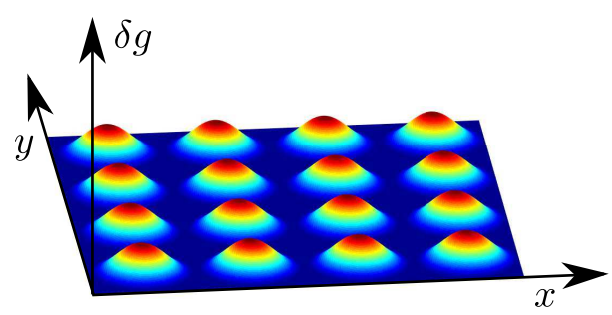

Figure 2. Curved medium with regularly arranged metric perturbations. The colors illustrate the strength of the perturbations, ranging from 0 (blue) to $a_{0}$ (red).

turbations, one might be inclined to simulate only one perturbation with periodic boundary conditions in all directions. However, since we put walls at $y=0, y=L_{y}$ and since we aim at extending this system to a random arrangement of perturbations, we simulate the entire regular system. In order to find the dependence of the flux on the perturbation amplitude $a_{0}$, range $r_{0}$, and density $n=N / V_{0}$, we have performed simulations in a wide parameter range. Our simulations range from dilute media $\left(r_{0}<\lambda\right)$, for which the metric perturbations are spatially separated from each other, to very dense media $\left(r_{0} \gg \lambda\right)$, where the metric perturbations overlap and form large clusters. As soon as the fluid reaches its equilibrium state, the average flux is measured according to Eq. (12). In Fig. 3 the flux deficit $\Delta \Phi$ is plotted against the metric parameters in a double-logarithmic plot. As can be seen, all the curves fulfill a power law with integer slopes, which is even valid in the regime of overlapping perturbations, $r_{0}>\lambda$. From the power law behavior, we can infer the following functional dependence of $\Delta \Phi$ on the metric parameters:

$$
\Delta \Phi=C_{0} a_{0} r_{0}^{2} n+\mathcal{O}\left(a_{0}^{2}\right),
$$

where $C_{0}$ is a constant. We recognize that this expression resembles the average metric perturbation, which we define as

$$
\langle\delta g\rangle=\frac{1}{V_{0}} \int \delta g d^{D} x
$$

where $d^{D} x=d x^{1} \cdots d x^{D}$. Plugging in the explicit expression for the radial perturbations (11) in two dimen- sions, we obtain

$$
\langle\delta g\rangle=-\frac{\pi^{2}-4}{8 \pi} a_{0} r_{0}^{2} n .
$$

Thus, we can rewrite Eq. (13) as follows:

$$
\langle\Phi\rangle=\left\langle\Phi_{0}\right\rangle \cdot\left(1+C_{1} \cdot\langle\delta g\rangle+\mathcal{O}\left(a_{0}^{2}\right)\right),
$$

where $C_{1}=1.500 \pm 0.003$. This relation describes the main contribution of the metric parameters to the flux. In order to check that relation Eq. (16) is not just a coincidence which only holds for this special type of radial perturbations, we have also studied metric perturbations of a different shape, given by the metric function

$$
\delta \widetilde{g}_{i}=\left\{\begin{array}{l}
-a_{0} \cos ^{2}\left(\pi \frac{\left|x-x_{i}\right|}{r_{0}}\right) \cos ^{2}\left(\pi \frac{\left|y-y_{i}\right|}{r_{0}}\right) \\
\text { if }\left|x-x_{i}\right| \leq \frac{r_{0}}{2},\left|y-y_{i}\right| \leq \frac{r_{0}}{2} \\
\text { else, }
\end{array}\right.
$$

where $\vec{r}_{i}=\left(x_{i}, y_{i}\right)$ denotes the center of the $i$ th perturbation. We will refer to this type as "square perturbations," as their support is a square, and mark all related quantities with a tilde symbol. For the square perturbations, the average metric perturbation is given by

$$
\langle\delta \widetilde{g}\rangle=-\frac{1}{4} a_{0} r_{0}^{2} n
$$

and only differs from the corresponding expression for the radial perturbations Eq. (15) in the constant prefactor.

Figure 4 shows the flux deficit $\Delta \Phi$ as function of the parameter combination $\left(a_{0} r_{0}^{2} n\right)$ for the two different types of metric perturbations. It can be seen that the flux in the simulations with square perturbations exhibits the same functional dependence on the metric parameters,

$$
\Delta \widetilde{\Phi}=\widetilde{C}_{0} a_{0} r_{0}^{2} n+\mathcal{O}\left(a_{0}^{2}\right),
$$

with a different slope $\widetilde{C}_{0}$. From the linear fits we obtain $C_{0}=0.350 \pm 0.004$ for the slope of the radial perturbations and $\widetilde{C}_{0}=0.374 \pm 0.003$ for the square perturbations. Assuming that relation Eq. (16) represents a universal flux law holding for both types of perturbations, the ratio between $\Delta \Phi$ and $\Delta \widetilde{\Phi}$ has to be equal to the ratio between $\langle\delta g\rangle$ and $\langle\delta \widetilde{g}\rangle$. We find:

$$
\begin{aligned}
& \frac{\Delta \widetilde{\Phi}}{\Delta \Phi}=\frac{\widetilde{C}_{0}}{C_{0}}=1.069 \pm 0.004, \\
& \frac{\langle\delta \widetilde{g}\rangle}{\langle\delta g\rangle}=\frac{2 \pi}{\pi^{2}-4}=1.070 .
\end{aligned}
$$

Thus, we conclude that — at least for the two types of metric perturbations considered here - relation Eq. (16) holds independently of the specific shape of the perturbations.

As already mentioned, relation Eq. (16) is expected to be only a linear approximation of the real functional 


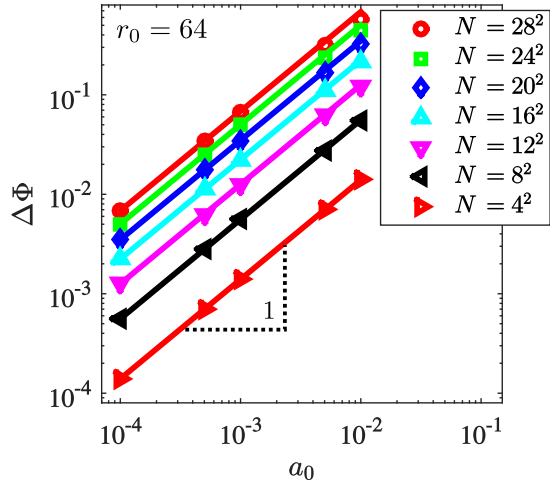

(a)

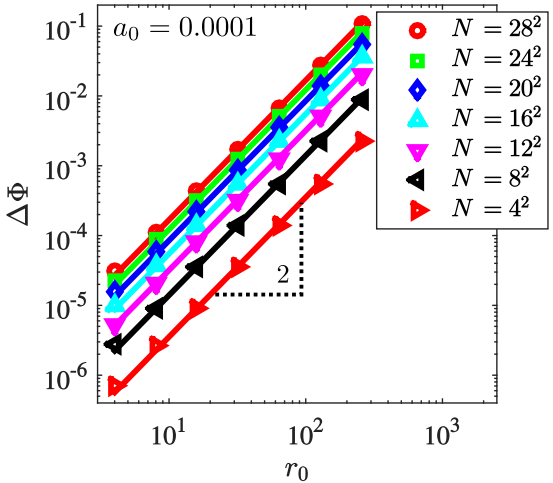

(b)

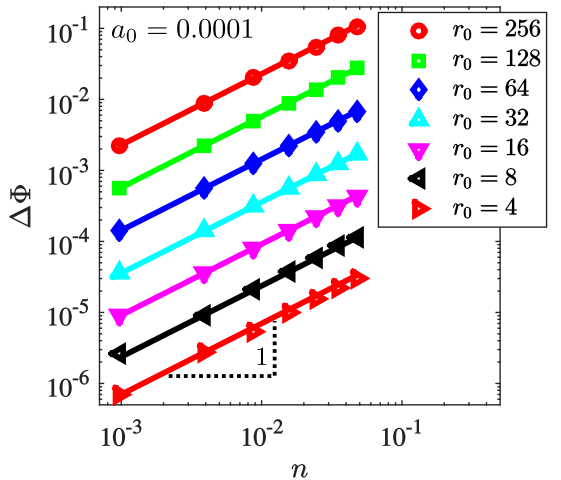

(c)

Figure 3. Flux deficit $\Delta \Phi=\frac{\left\langle\Phi_{0}\right\rangle-\langle\Phi\rangle}{\left\langle\Phi_{0}\right\rangle}$ as function of the metric perturbation amplitude $a_{0}$ (a), range $r_{0}$ (b), and density $n$ (c) for a $2 \mathrm{D}$ simulation with $N$ regularly arranged metric perturbations.

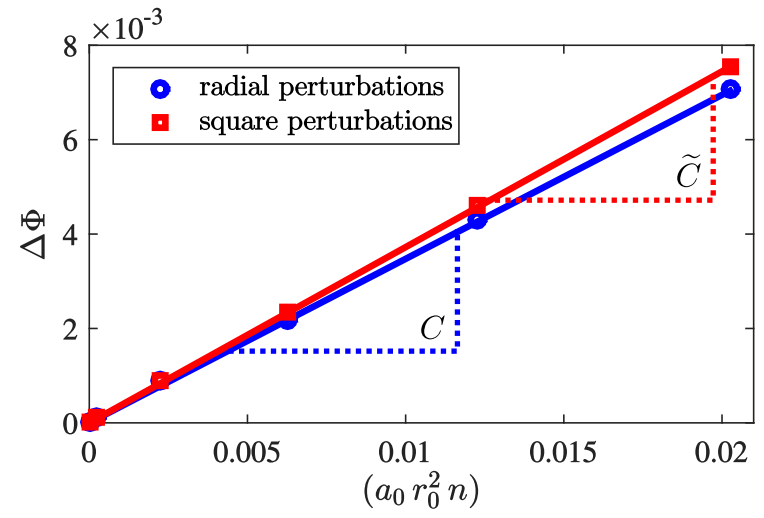

Figure 4. Flux deficit $\Delta \Phi=\frac{\left\langle\Phi_{0}\right\rangle-\langle\Phi\rangle}{\left\langle\Phi_{0}\right\rangle}$ as function of the product $a_{0} r_{0}^{2} n$ for two different types of metric perturbations. $a_{0}=0.001$ and $r_{0}=16$ are kept fixed while the number of perturbations is varied.

dependence of $\Phi$ on $\langle\delta g\rangle$. In order to find the higherorder contributions, we have also performed simulations for stronger spatial deformations, where non-linear effects are expected to appear. To this end, we have again varied all the metric parameters within a wide range of perturbation amplitudes $a_{0}$ and also included the case of negative amplitudes $a_{0}<0$. Figure 5 shows the resulting curve of $\langle\Phi\rangle /\left\langle\Phi_{0}\right\rangle$ as a function of $\langle\delta g\rangle$. As can be seen, all the points fall on one single curve, which leads to the conclusion that even beyond the first-order approximation in $a_{0}$, the flux is well-described as a function of $\langle\delta g\rangle$. From Fig. 5, we find the following flux law:

$$
\langle\Phi\rangle=\left\langle\Phi_{0}\right\rangle \cdot\left(1+\alpha^{2} \cdot\langle\delta g\rangle+2 \alpha^{2} \beta \cdot\langle\delta g\rangle^{2}\right),
$$

where $\alpha=1.223 \pm 0.001$ and $\beta=0.165 \pm 0.001$ are fitting parameters. This flux law is valid for metric perturbations $\langle\delta g\rangle=\mathcal{O}(1)$ and supports our expectations about transport in curved media: By adding a metric perturbation $\delta g$ to the flat space, the space becomes

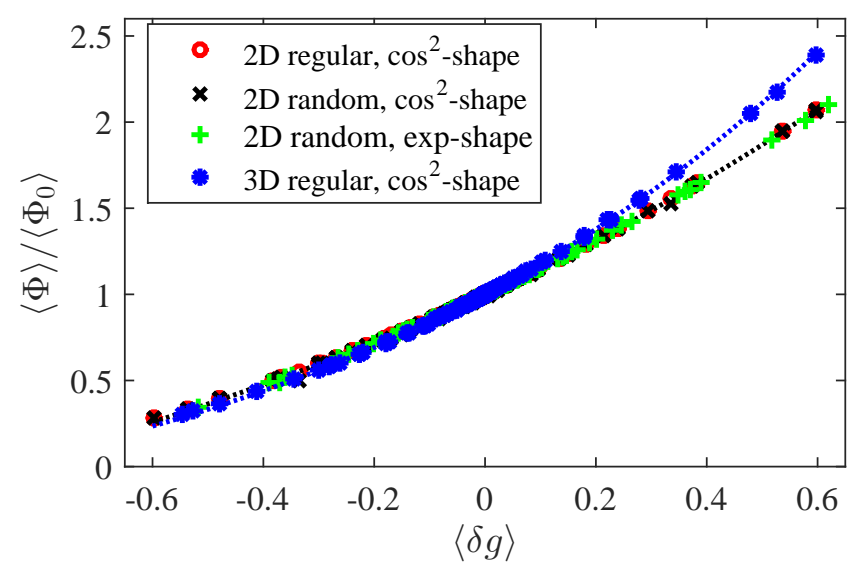

Figure 5. Flux ratio $\langle\Phi\rangle /\left\langle\Phi_{0}\right\rangle$ as function of the average strength of the metric perturbations $\langle\delta g\rangle$ for different types of curved media $(2 \mathrm{D}$ regular, $2 \mathrm{D}$ random and $3 \mathrm{D}$ regular with cos- or exp-shaped metric perturbations). For each medium type, all data points collapse onto a single common curve.

either stretched $(\delta g>0)$ or compressed $(\delta g<0)$. In the first case, $\delta g>0$, the cross-section of the medium is increased by the presence of the metric perturbations, such that the transport through the medium is enhanced and the flux increases compared to the flat space. For negative metric perturbations, $\delta g<0$, on the other hand, the channel cross-section is decreased, and the fluid transport deteriorates compared to the flat space.

We also have investigated the case of randomly distributed metric perturbations (see Fig. 6 for a sketch) in order to study the dependence of the Poiseuille flow on the spatial order of the metric perturbations. For small amplitudes $a_{0}$, the spatial arrangement of perturbations does not affect the linear order of the average metric perturbation, given by Eq. (15). Indeed, we find that the dependence of $\Delta \Phi$ on the metric parameters is exactly the same as in the regular case, given by Eq. (13), and 


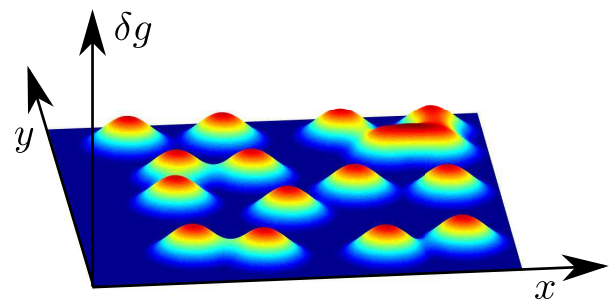

Figure 6. Curved medium with randomly arranged metric perturbations. The colors illustrate the strength of the metric perturbation, ranging from 0 (blue) to $a_{0}$ (red).

the flux is thus well-described as a function of $\langle\delta g\rangle$. Even for larger amplitudes $a_{0}$, the flux curve does not change notably from the case of regularly arranged metric perturbations, as can be seen in Fig. 5. We find that the flux follows Eq. (18) with fitting parameters $\alpha=1.224 \pm 0.002$ and $\beta=0.158 \pm 0.005$, in agreement with the corresponding values for regularly arranged metric perturbations. Thus, we conclude that - within the range of parameters studied - the spatial order of the metric perturbations does not affect the behavior of the flux significantly.

In order to check that the flux law also holds for metric perturbations with non-compact support, we have also performed simulations for exponentially shaped perturbations of the form $\delta g_{i}=-a_{0} \exp \left(-r_{i} / r_{0}\right)$, distributed randomly in space. For this perturbation type, the average metric perturbation yields $\langle\delta g\rangle=-2 \pi a_{0} r_{0}^{2} n$, and the simulations results have been added to Fig. 5 with fitting coefficients $\alpha=1.225 \pm 0.001$ and $\beta=0.144 \pm 0.002$. As can be seen, the flux curve coincides with the other flux curves in 2D, which supports our claim that the flux law Eq. (18) holds universally.

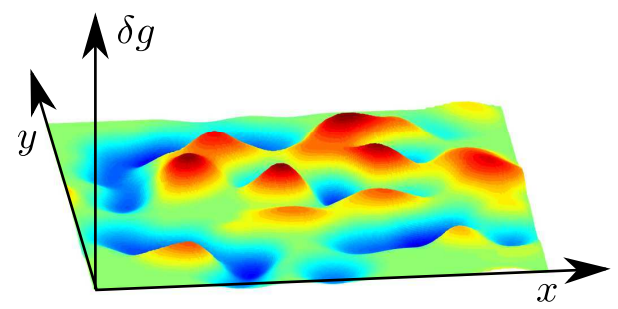

Figure 7. Curved medium with mixed positive and negative metric perturbations. The colors illustrate the strength of the metric perturbation, ranging from $-a_{0}$ (blue) to $+a_{0}$ (red).

Additionally, we have also simulated media with both positive and negative metric perturbations (see Fig. 7 for a sketch). If the flux law Eq. (18) holds in general, any non-trivial configuration of perturbations with $\langle\delta g\rangle=0$ should give the same value for the flux, namely $\langle\Phi\rangle=\left\langle\Phi_{0}\right\rangle$. To test this statement, we have performed simulations for a wide range of metric parameters, $a_{0} \in\{ \pm 0.0001, \pm 0.001, \pm 0.01\}, r_{0} \in$ $\{4,8,16,32,64,128\}, N \in\{16,64\}$, all satisfying $\langle\delta g\rangle=$ 0 . Indeed, we find that all simulations give the same flux $\Phi_{0}$ with a standard deviation of about $0.2 \%$.

\section{B. 3D curved media}

Next, we consider a three-dimensional curved channel equipped with $N$ regularly arranged metric perturbations at positions

$$
\vec{r}_{n}=\vec{r}_{n_{x}, n_{y}, n_{z}}=\left(n_{x}, n_{y}, n_{z}\right) \cdot \lambda, \quad n_{x}, n_{y}, n_{z} \in \mathbb{Z},
$$

where $\lambda=\sqrt[3]{V_{0} / N}$ denotes the characteristic distance between the metric perturbations and $V_{0}=$ $L_{x} L_{y} L_{z} \Delta x \Delta y \Delta z$ is the volume of the flat background space.

Like for the two-dimensional cases, we have studied the dependence of the Poiseuille flow on the metric parameters as depicted in Fig. 8. We find that - at leading order in the metric perturbations - the flux deficit is given by

$$
\Delta \Phi=C_{0} a_{0} r_{0}^{3} n+\mathcal{O}\left(a_{0}^{2}\right),
$$

$C_{0}$ being a constant. In analogy to the two-dimensional case, the expression $\left(a_{0} r_{0}^{3} n\right)$ corresponds to the average metric perturbation in three dimensions, given by

$$
\langle\delta g\rangle=-\frac{\pi^{2}-6}{12 \pi} a_{0} r_{0}^{3} n .
$$

Plotting the flux versus $\langle\delta g\rangle$ for $|\langle\delta g\rangle| \leq 0.6$, as shown in Fig. 5, we again find that even beyond the leading order, all points fall on one single curve, which can be described by the flux law

$$
\langle\Phi\rangle=\left\langle\Phi_{0}\right\rangle \cdot\left(1+\alpha^{3} \cdot\langle\delta g\rangle+3 \alpha^{3} \beta \cdot\langle\delta g\rangle^{2}\right),
$$

where the fitting parameters take the values $\alpha=1.21 \pm$ 0.01 and $\beta=0.17 \pm 0.01$ and thus agree with the corresponding values of the two-dimensional flux law Eq. (18). This suggests that the flux law might be generalized as follows:

$$
\langle\Phi\rangle=\left\langle\Phi_{0}\right\rangle \cdot\left(1+\alpha^{D} \cdot\langle\delta g\rangle+D \alpha^{D} \beta \cdot\langle\delta g\rangle^{2}\right),
$$

where $D$ denotes the dimension of the system and universal constants $\alpha \approx 1.22$ and $\beta \approx 0.16$.

\section{COMPARISON WITH PREVIOUS STUDY}

The findings presented in this paper generalize the results of the previous study [24] of three-dimensional curved media equipped with randomly distributed metric perturbations of exponential shape, $\delta g_{i}=$ $-a_{0} \exp \left(-r_{i} / r_{0}\right)$. In the previous study, only positive amplitudes (corresponding to negative values of $\langle\delta g\rangle$ ) were considered, and the flux was found to obey the laws

$$
\begin{aligned}
& \Phi_{0}-\Phi=A_{0} \frac{N / N_{0}}{1+\left(N / N_{0}\right)^{2}}, \\
& \Phi_{0}-\Phi=A_{0} \frac{\left(\epsilon / \epsilon_{0}\right)^{3}}{1+\left(\epsilon / \epsilon_{0}\right)^{6}}
\end{aligned}
$$




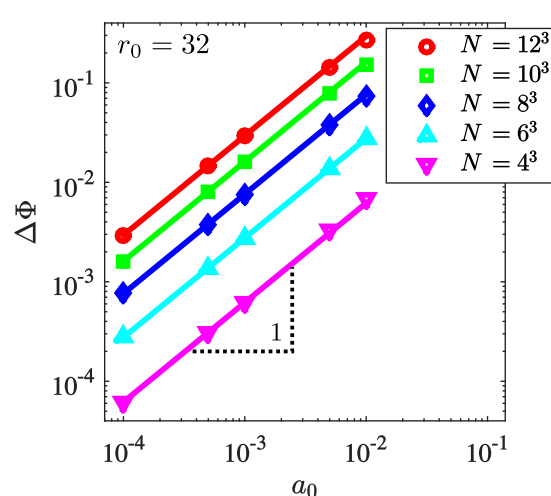

(a)

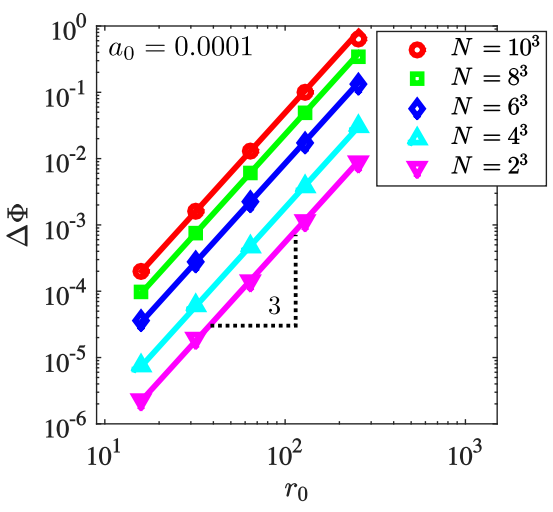

(b)

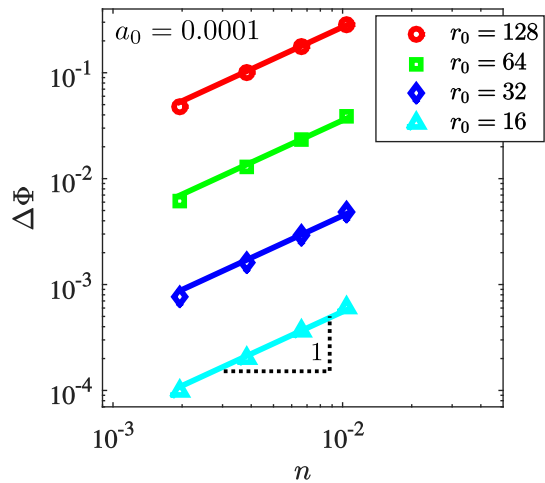

(c)

Figure 8. Flux deficit $\Delta \Phi=\frac{\left\langle\Phi_{0}\right\rangle-\langle\Phi\rangle}{\left\langle\Phi_{0}\right\rangle}$ as function of the metric perturbation amplitude $a_{0}$ (a), range $r_{0}$ (b), and density $n$ (c) for a $3 \mathrm{D}$ simulation with regularly arranged metric perturbations.

where $N$ denotes the number of metric perturbations, $\epsilon=r_{0} / \lambda$ is the dimensionless deformation, $\lambda=\sqrt[3]{V_{0} / N}$ is the characteristic length, $V_{0}$ denotes the volume of the flat background space, and $A_{0}, N_{0}$, and $\epsilon_{0}$ are fitting parameters. Calculating the average metric perturbation for this perturbation type, we obtain

$$
\langle\delta g\rangle=-8 \pi a_{0} r_{0}^{3} n=-8 \pi a_{0} \epsilon^{3} .
$$

With that, Eqs. (21) and (22) can be combined into a single equation of the following form:

$$
\frac{\langle\Phi\rangle}{\left\langle\Phi_{0}\right\rangle} \approx 1+\widetilde{A}_{0} \frac{\langle\delta g\rangle /\langle\delta g\rangle_{0}}{1+\left(\langle\delta g\rangle /\langle\delta g\rangle_{0}\right)^{2}}
$$

where $\widetilde{A}_{0}$ and $\langle\delta g\rangle_{0}$ are constants. Here, we have switched to the spatially averaged flux, dividing by the cross-sectional area $S$ according to Eq. (12).

Restricting to positive amplitudes, i.e., $\langle\delta g\rangle<0$, the flux curves depicted in Fig. 5 are indeed well-described by Eq. (23), thus showing that the flux law in Ref. [24] is consistent with our present results. However, Eq. (23) fails to describe the correct behavior for negative amplitudes (corresponding to $\langle\delta g\rangle>0$ ) and should thus be replaced by the more general flux law given by Eq. (20). This flux law also explains the power law dependence of the flux deficit on $\epsilon$ for small $a_{0}$ found in Ref. [24], since at leading order in $a_{0}$ we have $\left(\left\langle\Phi_{0}\right\rangle-\langle\Phi\rangle\right) \sim\langle\delta g\rangle \sim$ $r_{0}^{3} n=\epsilon^{3}$.

\section{METHOD VALIDATION}

\section{A. Improvement by Cancelation of Discrete Lattice Effects}

In this section we validate the improvement of our method by the cancelation of discrete lattice effects, which arise from the forcing term of the lattice
Boltzmann equation. As already mentioned in Sec. II B, discrete lattice effects manifest themselves as spurious source terms of order $\Delta t$ in the Navier-Stokes equations [see Eq. (5)], which - depending on the strength of the spatial deformation - have a considerable effect on the fluid. To illustrate this effect, we have performed twodimensional simulations with and without the spurious source terms of order $\Delta t$ for a curved medium with 8 metric perturbations of amplitude $a_{0}=0.1$ and range $r_{0}=22$. The fluid is driven through the medium by an external force corresponding to a pressure drop of $\nabla P=10^{-6}$ in $x$ direction, and the relaxation time is set to $\tau=1$. Figure 9 shows the velocity field of the fluid for a simulation with discrete lattice effects (left) and the same simulation using the corrected method (right). In

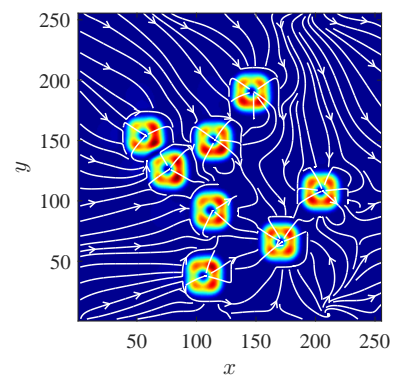

(a)

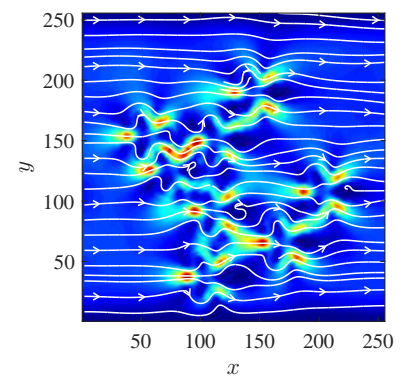

(b)
Figure 9. Velocity streamlines of the fluid for a simulation with $N=8$ metric perturbations with amplitude $a_{0}=0.1$ and range $r_{0}=22$. The colors illustrate the absolute value of the velocity. (a) Simulation with discrete lattice effects and spurious source terms. (b) Corrected model.

the left figure, one can clearly see large unphysical sinks in the velocity field, originating from the spurious source terms in the Navier-Stokes equations. By properly correcting the density and velocity fields in each time step as described in Sec. II B, these unphysical source terms 
are eliminated at order $\Delta t$, leading to the source-free velocity field depicted in the right figure, which appears much more physical. The improvement becomes even more obvious in the corresponding divergence field of the velocity, which is plotted as a function of $x$ in Fig. 10 at the cross-section $y=L_{y} / 2$. According to the continuity

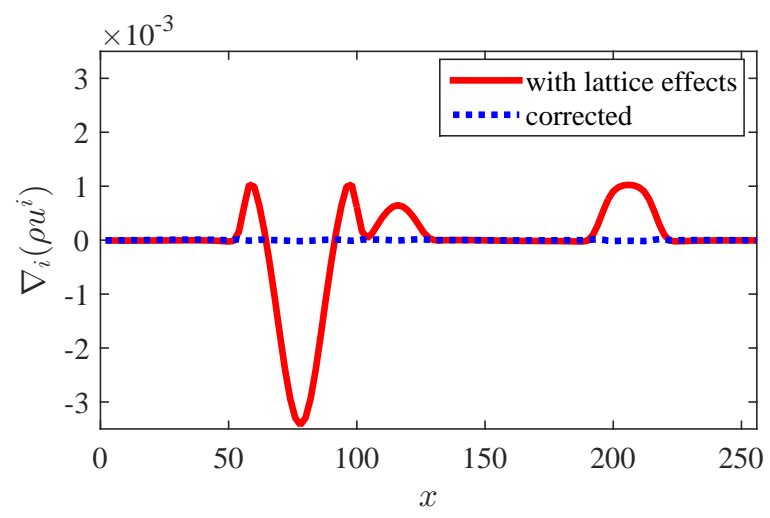

Figure 10. The divergence field $\nabla_{i}\left(\rho u^{i}\right)$ as a function of $x$ at the cross-section $y=L_{y} / 2$ for the model with spurious lattice effects and for the corrected model, in which discrete lattice effects are canceled at order $\Delta t$.

Eq. (2), $\nabla_{i}\left(\rho u^{i}\right)$ must vanish identically in the stationary state, which is clearly fulfilled for the corrected method, whereas the uncorrected method causes large deviations due to the unphysical source terms.

\section{B. Finite resolution study}

In order to investigate the effect of the grid resolution on our data we have compared simulations of the twodimensional curved medium described in Sec. III A) for different grid resolutions $\Delta x$, corresponding to different system lengths $L=128 / \Delta x$. Figure 11 depicts the corresponding flux curves for system lengths $L=64,128$, and 192, where we abbreviate the flux ratio at system length $L$ by $f_{L}:=\langle\Phi\rangle /\left\langle\Phi_{0}\right\rangle$. As can be seen, for increas-

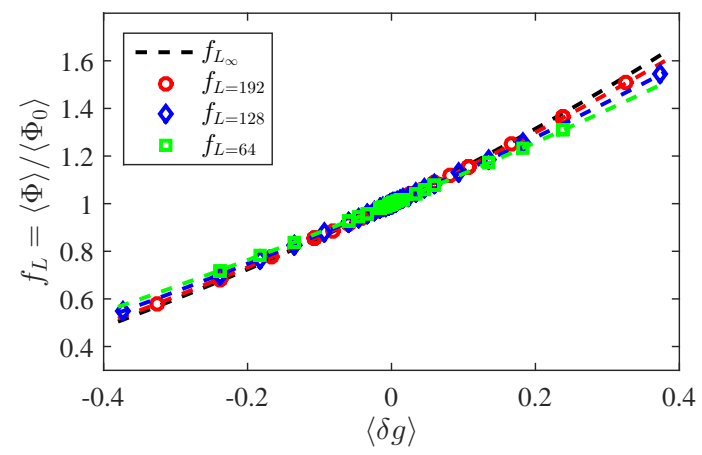

Figure 11. Flux ratio $\langle\Phi\rangle /\left\langle\Phi_{0}\right\rangle$ as function of $\langle\delta g\rangle$ for different system sizes.

ing system lengths $L$ the flux curves $f_{L}$ converge rapidly to the limiting function $f_{L_{\infty}}$, which we approximate by the flux law given by Eq. (18) for $L=256$. In order to prove the convergence quantitatively, we show that the relative distance between the curves, defined as

$$
\Delta_{L}^{2}:=\frac{\left\|f_{L}-f_{L_{\infty}}\right\|^{2}}{\left\|f_{L_{\infty}}\right\|^{2}}=\frac{\int\left|f_{L}(x)-f_{L_{\infty}}(x)\right|^{2} d x}{\int\left|f_{L_{\infty}}(x)\right|^{2} d x},
$$

converges to zero with increasing system size $L^{2}$. Figure 12 depicts the relative distance $\Delta_{L}$ as function of the system size in a semi-logarithmic plot. As can be seen,

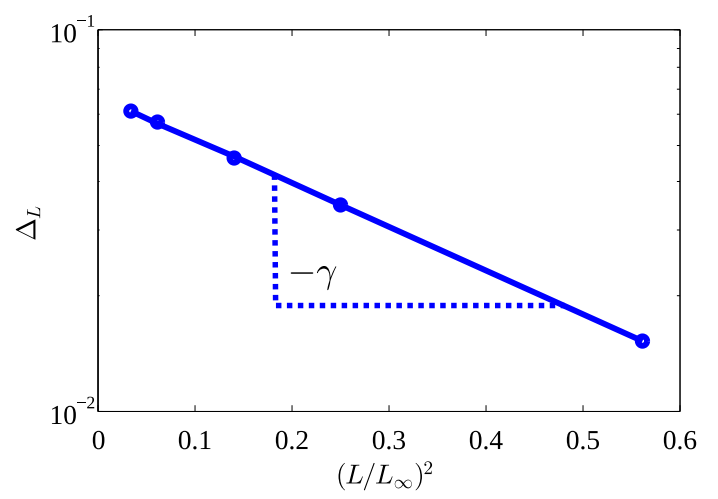

Figure 12. Relative distance between the flux curves as function of the system size $L^{2}$.

the flux curves $f_{L}$ converge to $f_{L_{\infty}}$ exponentially with $L^{2}$,

$$
\Delta_{L} \sim \exp \left(-\gamma \frac{L^{2}}{L_{\infty}^{2}}\right)
$$

with $\gamma=2.66 \pm 0.05$. Consequently, we conclude that within an error of about 1\% - our numerical results are free of finite resolution effects.

\section{Flux conservation}

In order to check to which extent the flux is numerically conserved, it is insightful to plot the profiles $\Phi(x)$ along the channel direction $x$. In Fig. 13, the relative fluctuation of the flux around its mean value, $\Delta_{x}:=\left(\Phi-\langle\Phi\rangle_{x}\right) /\langle\Phi\rangle_{x}$, is plotted versus $x$ for different randomly curved media. As can be seen, the flux varies only marginally with $x$, with an average standard deviation of $\Delta_{x} \approx 2 \cdot 10^{-5}$. Thus, we conclude that the flux is numerically conserved up to a negligible error of about $0.002 \%$.

The conservation of the flux is closely connected to the improvements of the method depicted in Fig. 10, since the flux loss between two channel cross-sections $x=x_{1}$ and $x=x_{2}$ is proportional to the divergence of $\left(\rho u^{i}\right)$ :

$$
\Phi\left(x_{2}\right)-\Phi\left(x_{1}\right)=\oint_{\partial V} g(\rho \vec{u}, \vec{n}) d(\partial V)=\int_{V} \nabla_{i}(\rho u)^{i} d V,
$$




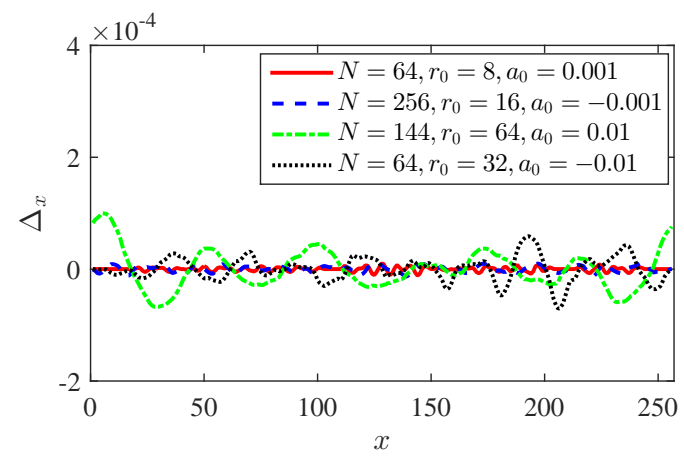

Figure 13. Relative fluctuation of the flux, $\Delta_{x}=(\Phi-$ $\left.\langle\Phi\rangle_{x}\right) /\langle\Phi\rangle_{x}$, as function of $x$.

where $V$ denotes the volume between the channel crosssections. Here, we have used Gauss' integration theorem and the fact that the flux vanishes at the walls.

\section{CONCLUSIONS}

In this paper, we have studied Poiseuille flow in curved space, using the lattice Boltzmann method in curved space to simulate the flow according to the covariant Navier-Stokes equations. We have established a relation between the flux of the Poiseuille flow in the stationary state and the metric parameters of the medium (e.g., amplitude $a_{0}$, range $r_{0}$, and density $n$ of the metric perturbations) for different types of curved media, starting from two-dimensional media with regularly and randomly arranged metric perturbations and ending with three-dimensional media. In all cases, we have found that the flux depends only on a specific combination of parameters, which we have identified as the average metric perturbation,

$$
\langle\delta g\rangle \sim a_{0} r_{0}^{D} n,
$$

where $D$ denotes the dimension of the medium. Even beyond leading order in the metric perturbations, the flux is well-described as a function of the average metric perturbation $\langle\delta g\rangle$. Explicitly, we have found that the (spatially averaged) flux $\langle\Phi\rangle$ is well-described by the following flux law:

$$
\langle\Phi\rangle=\left\langle\Phi_{0}\right\rangle \cdot\left(1+\alpha^{D} \cdot\langle\delta g\rangle+D \alpha^{D} \beta \cdot\langle\delta g\rangle^{2}\right),
$$

where $\left\langle\Phi_{0}\right\rangle$ denotes the (spatially averaged) flux in flat space and $\alpha$ and $\beta$ are fitting parameters. This relation is universal in the sense that - within the parameter range considered here - it depends neither on the specific shape nor on the spatial order of the metric perturbations and is valid both for two- and three-dimensional systems.

For the simulations, we have improved our curvedspace lattice Boltzmann method further by canceling discrete lattice effects, which originate from the forcing term, at order $\Delta t$. These discrete lattice effects appear as spurious source terms in the Navier-Stokes equations and thus reduce the accuracy of the method. In curved spaces, the cancelation of spurious terms turns out to be a sophisticated task since the inertial forces depend quadratically on the fluid velocity and even contribute to the continuity equation. Still, it has been possible to eliminate all spurious terms of order $\Delta t$ by correcting not only the fluid velocity at each time step (as is commonly suggested in the literature [27]), but also the fluid density. With the corrected model at hand, we have been able to improve the accuracy of the results considerably.

\section{Appendix A: Cancelation of Discrete Lattice Effects: A Chapman-Enskog Analysis}

In the following derivation, we will use the abbreviations

$\overline{\sum_{\lambda}}:=\sum_{\lambda} \sqrt{g} \quad, \quad D_{t}:=\partial_{t}+c_{\lambda}^{i} \partial_{i} \quad, \quad \bar{\partial}_{i}:=\partial_{i}-\Gamma_{i j}^{j}$.

\section{Ansatz and preparation}

We show that spurious discrete lattice effects originating from the forcing term can be canceled at first order in $\Delta t$ by a redefinition of density $\rho \rightarrow \hat{\rho}$ and velocity $u^{i} \rightarrow \hat{u}^{i}$. Inspired by Refs. [27, 31], we make the following ansatz for the corrected quantities $\hat{\rho}$ and $\hat{u}^{i}$ :

$$
\begin{gathered}
\hat{\rho}=\rho+\Delta t R(\hat{\rho}, \hat{u})=\overline{\sum_{\lambda}} f_{\lambda}+\Delta t R(\hat{\rho}, \hat{u}), \quad(\mathrm{A} 1) \\
\hat{\rho} \hat{u}^{i}=\rho u^{i}+\Delta t U^{i}(\hat{\rho}, \hat{u})=\overline{\sum_{\lambda}} f_{\lambda} c_{\lambda}^{i}+\Delta t U^{i}(\hat{\rho}, \hat{u}),
\end{gathered}
$$

where $R(\hat{\rho}, \hat{u})$ and $U^{i}(\hat{\rho}, \hat{u})$ are correction functions whose explicit form has to be determined in such a way that discrete lattice effects are canceled in the Navier-Stokes equations. The corrected quantities $\hat{\rho}$ and $\hat{u}^{i}$ are determined implicity by Eqs. (A1) and (A2). This system of equations can be solved for $\hat{\rho}$ and $\hat{u}^{i}$ by using standard numerical techniques (e.g., Newtons algorithm, see Appendix A 6). To find the explicit expressions for the correction functions $R(\hat{\rho}, \hat{u})$ and $U^{i}(\hat{\rho}, \hat{u})$, we perform a Chapman-Enskog expansion of the lattice Boltzmann equation in curved space,

$f_{\lambda}\left(x^{i}+c_{\lambda}^{i} \Delta t, t+\Delta t\right)-f_{\lambda}\left(x^{i}, t\right)=-\frac{1}{\tau}\left(f_{\lambda}-f_{\lambda}^{\mathrm{eq}}\right)+\Delta t \mathcal{F}_{\lambda}$.

The equilibrium distribution as well as the forcing term are evaluated in terms of the corrected quantities $\hat{\rho}$ and $\hat{u}$, i.e., $f_{\lambda}^{\text {eq }}=f_{\lambda}^{\text {eq }}(\hat{\rho}, \hat{u}), \mathcal{F}_{\lambda}=\mathcal{F}_{\lambda}(\hat{\rho}, \hat{u})$. Both are expanded 
into tensor Hermite polynomials,

$$
\begin{aligned}
& \mathcal{H}_{(0)}=1, \quad \mathcal{H}_{(1)}^{i}=\frac{c_{\lambda}^{i}}{c_{s}}, \quad \mathcal{H}_{(2)}^{i j}=\frac{c_{\lambda}^{i} c_{\lambda}^{j}}{c_{s}^{2}}-\delta^{i j}, \\
& \mathcal{H}_{(3)}^{i j k}=\frac{c_{\lambda}^{i} c_{\lambda}^{j} c_{\lambda}^{k}}{c_{s}^{3}}-\left(\delta^{i j} \frac{c_{\lambda}^{k}}{c_{s}}+\delta^{j k} \frac{c_{\lambda}^{i}}{c_{s}}+\delta^{k i} \frac{c_{\lambda}^{j}}{c_{s}}\right) .
\end{aligned}
$$

This yields (up to second order):

$$
f_{\lambda}^{\mathrm{eq}}=\frac{w_{\lambda}}{\sqrt{g}} \sum_{n=0}^{3} \frac{1}{n ! c_{s}^{n}} a_{(n)}^{\mathrm{eq}, I_{n}} \mathcal{H}_{(n)}^{I_{n}},
$$

where $I_{n}=\left(i_{1}, \ldots, i_{n}\right)$ denotes an index $n$-tuple, which is summed over. The expansion coefficients are given by

$$
\begin{aligned}
& a_{(0)}^{\mathrm{eq}}=\hat{\rho}, \quad a_{(1)}^{\mathrm{eq}, i}=\hat{\rho} \hat{u}^{i}, \quad a_{(2)}^{\mathrm{eq}, i j}=\hat{\rho} c_{s}^{2} \Delta^{i j}+\hat{\rho} \hat{u}^{i} \hat{u}^{j}, \\
& a_{(3)}^{\mathrm{eq}, i j k}=\hat{\rho} c_{s}^{2}\left(\Delta^{i j} \hat{u}^{k}+\Delta^{j k} \hat{u}^{i}+\Delta^{k i} \hat{u}^{j}\right)+\hat{\rho} \hat{u}^{i} \hat{u}^{j} \hat{u}^{k},
\end{aligned}
$$

where $\Delta^{i j}:=g^{i j}-\delta^{i j}$ is a measure for the deviation from flat space. The forcing term reads

$$
\mathcal{F}_{\lambda}=\frac{w_{\lambda}}{\sqrt{g}} \sum_{n=0}^{2} \frac{1}{n ! c_{s}^{n+1}} a_{(n)}^{I_{n}} F_{\lambda}^{i_{n+1}} \mathcal{H}_{(n+1)}^{I_{n+1}},
$$

with coefficients

$$
a_{(0)}=a_{(0)}^{\mathrm{eq}}, \quad a_{(1)}^{i}=a_{(1)}^{\mathrm{eq}, i}, \quad a_{(2)}^{i j}=a_{(2)}^{\mathrm{eq}, i j}-\sigma^{i j} .
$$

Here, $F_{\lambda}^{i}=-\Gamma_{j k}^{i} c_{\lambda}^{j} c_{\lambda}^{k}$ represents inertial forces and $\sigma^{i j}=$ $-\left(1-\frac{1}{2 \tau}\right) \bar{\sum}_{\lambda} c_{\lambda}^{i} c_{\lambda}^{j}\left(f_{\lambda}-f_{\lambda}^{\mathrm{eq}}\right)$ is the viscous stress tensor.

From Eqs. (A4) and (A5), we can calculate the moments of $f_{\lambda}^{\text {eq }}$ and $\mathcal{F}_{\lambda}$, which are needed for the ChapmanEnskog expansion later. For this purpose, we apply the orthogonality condition of the Hermite polynomials, which is supported by the D3Q41 lattice up to third order,

$$
\sum_{\lambda} w_{\lambda} \mathcal{H}_{(n)}^{I_{n}} \mathcal{H}_{(m)}^{J_{m}}=\delta_{n m} \delta^{I_{n} J_{n}}, \quad 0 \leq n, m \leq 3,
$$

where $I_{n}=\left(i_{1}, \ldots, i_{n}\right), J_{m}=\left(j_{1}, \ldots, j_{m}\right)$ are index tupels and $\delta^{I_{n} J_{n}}:=\sum_{\sigma \in S_{n}}\left(\delta^{i_{1} j_{\sigma(1)}} \cdots \delta^{i_{n} j_{\sigma(n)}}\right)$.

This yields:

$$
\begin{aligned}
\hat{\rho} & =\overline{\sum_{\lambda}} f_{\lambda}^{\mathrm{eq}}, \\
\hat{\rho} \hat{u}^{i} & =\overline{\sum_{\lambda}} f_{\lambda}^{\mathrm{eq}} c_{\lambda}^{i}, \\
\Pi^{\mathrm{eq}, i j} & =\overline{\sum_{\lambda}} f_{\lambda}^{\mathrm{eq}} c_{\lambda}^{i} c_{\lambda}^{j}=\hat{\rho}\left(c_{s}^{2} g^{i j}+\hat{u}^{i} \hat{u}^{j}\right), \\
\Sigma^{\mathrm{eq}, i j k} & =\overline{\sum_{\lambda}} f_{\lambda}^{\mathrm{eq}} c_{\lambda}^{i} c_{\lambda}^{j} c_{\lambda}^{k}=\hat{\rho} c_{s}^{2}\left(\hat{u}^{i} g^{j k}+\hat{u}^{j} g^{i k}+\hat{u}^{k} g^{i j}\right) \\
& +\mathcal{O}\left(\hat{u}^{3}\right)
\end{aligned}
$$

Here, we have defined $T^{i j}:=\Pi^{\mathrm{eq}, i j}-\sigma^{i j}$ and neglected terms of order $\mathcal{O}\left(\hat{u}^{3}\right) \sim \mathcal{O}\left(\mathrm{Ma}^{3}\right)$ in $C^{i j}$, where Ma denotes the Mach number.

\section{Multiscale Expansion}

We start with the Chapman-Enskog expansion by expanding the distribution function and the time derivative in the Knudsen number $\varepsilon$ :

$$
\begin{aligned}
f & =f^{(0)}+\varepsilon f^{(1)}+\varepsilon^{2} f^{(2)}+\ldots, \\
\partial_{t} & =\varepsilon \partial_{t}^{(1)}+\varepsilon^{2} \partial_{t}^{(2)}+\ldots,
\end{aligned}
$$

Furthermore, we rescale all other quantities $Q=$ $\left(\partial_{i}, \nabla_{i}, \Gamma_{j k}^{i}, \mathcal{F}, A, B^{i}, R, U^{i}, \sigma^{i j}, \ldots\right)$ by the Knudsen number, $Q=\varepsilon Q^{(1)}$.

Plugging everything into Eq. (A3) and comparing orders of $\varepsilon$, we obtain the following equations:

$$
\begin{array}{ll}
\mathcal{O}\left(\varepsilon^{0}\right): & f_{\lambda}^{(0)}=f_{\lambda}^{\mathrm{eq}}, \\
\mathcal{O}\left(\varepsilon^{1}\right): & D_{t}^{(1)} f_{\lambda}^{(0)}=-\frac{1}{\tau \Delta t} f_{\lambda}^{(1)}+\mathcal{F}_{\lambda}^{(1)}, \\
\mathcal{O}\left(\varepsilon^{2}\right): & \partial_{t}^{(2)} f_{\lambda}^{(0)}+\left(1-\frac{1}{2 \tau}\right) D_{t}^{(1)} f_{\lambda}^{(1)} \\
& =-\frac{1}{\tau \Delta t} f_{\lambda}^{(2)}-\frac{\Delta t}{2} D_{t}^{(1)} \mathcal{F}_{\lambda}^{(1)} .
\end{array}
$$

Comparing Eqs. (A1) and (A2) to Eqs. (A6) and (A7) with $f_{\lambda}^{(0)}=f_{\lambda}^{\text {eq }}$, we see that

$$
\bar{\sum}_{\lambda} f_{\lambda}^{(1)}=-\Delta t R^{(1)}, \quad \bar{\sum}_{\lambda} f_{\lambda}^{(1)} c_{\lambda}^{i}=-\Delta t U^{(1), i} .
$$

\section{Moments of Eqs. (A14) and (A15)}

Taking the moments of Eq. (A14) yields:

$$
\bar{\sum}_{\lambda}\left[\text { Eq. (A14)] : } \partial_{t}^{(1)} \hat{\rho}+\bar{\partial}_{i}^{(1)}\left(\hat{\rho} \hat{u}^{i}\right)=A^{(1)}+\frac{1}{\tau} R^{(1)},\right.
$$

$\bar{\sum}_{\lambda} c_{\lambda}^{i}\left[\right.$ Eq. (A14)] $: \partial_{t}^{(1)}\left(\hat{\rho} \hat{u}^{i}\right)+\bar{\partial}_{j}^{(1)} \Pi^{(0), i j}=B^{(1), i}+\frac{1}{\tau} U^{(1), i}$,

where $\Pi^{(0), i j}=\Pi^{\mathrm{eq}, i j}=\hat{\rho}\left(c_{s}^{2} g^{i j}+\hat{u}^{i} \hat{u}^{j}\right)$, and the $\bar{\partial}_{i}$ derivative originates from

$$
\begin{aligned}
\sum_{\lambda} \sqrt{g} c_{\lambda}^{i} \partial_{i} f_{\lambda}^{(0)} & =\partial_{i} \sum_{\lambda} \sqrt{g} c_{\lambda}^{i} f_{\lambda}^{(0)}-\sum_{\lambda}\left(\partial_{i} \sqrt{g}\right) c_{\lambda}^{i} f_{\lambda}^{(0)} \\
& =\partial_{i}\left(\hat{\rho} \hat{u}^{i}\right)-\Gamma_{i j}^{j}\left(\hat{\rho} \hat{u}^{i}\right)=: \bar{\partial}_{i}\left(\hat{\rho} \hat{u}^{i}\right)
\end{aligned}
$$


where we have used the identity $\partial_{i} \sqrt{g}=\Gamma_{i j}^{j} \sqrt{g}$. The moments of Eq. (A15) are given by

$$
\begin{aligned}
& \bar{\sum}_{\lambda}[\text { Eq. (A15)] : } \\
& \partial_{t}^{(2)} \hat{\rho}=\Delta t\left(1-\frac{1}{2 \tau}\right)\left(\partial_{t}^{(1)} R^{(1)}+\bar{\partial}_{i}^{(1)} U^{(1), i}\right) \\
& -\frac{\Delta t}{2}\left(\partial_{t}^{(1)} A^{(1)}+\bar{\partial}_{i}^{(1)} B^{(1), i}\right),
\end{aligned}
$$

$\bar{\sum}_{\lambda} c_{\lambda}^{i}[$ Eq. $(\mathrm{A} 15)]:$

$$
\begin{array}{r}
\partial_{t}^{(2)}\left(\hat{\rho} \hat{u}^{i}\right)=\bar{\partial}_{j}^{(1)} \sigma^{(1), i j}-\frac{\Delta t}{2} \partial_{t}^{(1)} B^{(1), i} \\
+\Delta t\left(1-\frac{1}{2 \tau}\right) \partial_{t}^{(1)} U^{(1), i}
\end{array}
$$

where $\sigma^{(1), i j}$, the viscous stress tensor (rescaled by $\varepsilon$ ), is defined as

$$
\sigma^{(1), i j}=-\left(1-\frac{1}{2 \tau}\right) \bar{\sum}_{\lambda} f_{\lambda}^{(1)} c_{\lambda}^{i} c_{\lambda}^{j} .
$$

The explicit expression for the viscous stress tensor will be derived later.

\section{Continuity Equation}

For the continuity equation, we add $\varepsilon[$ Eq. (A17)] and $\varepsilon^{2}[$ Eq. (A19)]:

$$
\begin{aligned}
\partial_{t} \hat{\rho}+\bar{\partial}_{i}\left(\hat{\rho} \hat{u}^{i}\right)= & A+\frac{1}{\tau} R-\frac{\Delta t}{2}\left(\partial_{t} A+\bar{\partial}_{i} B^{i}\right) \\
& +\Delta t\left(1-\frac{1}{2 \tau}\right)\left(\partial_{t} R+\bar{\partial}_{i} U^{i}\right) .
\end{aligned}
$$

In order to cancel all spurious terms of order $\Delta t$, we first rescale the forcing term by a factor of $\left(1-\frac{1}{2 \tau}\right)$,

$$
\mathcal{F}_{\lambda} \rightarrow\left(1-\frac{1}{2 \tau}\right) \mathcal{F}_{\lambda}
$$

which implies $\left(A, B^{i}\right) \rightarrow\left(1-\frac{1}{2 \tau}\right)\left(A, B^{i}\right)$. With this redefinition, Eq. (A22) becomes

$$
\begin{aligned}
& \quad \partial_{t} \hat{\rho}+\bar{\partial}_{i}\left(\hat{\rho} \hat{u}^{i}\right)=\left(1-\frac{1}{2 \tau}\right) A+\frac{1}{\tau} R \\
& +\Delta t\left(1-\frac{1}{2 \tau}\right)\left(\partial_{t}\left(R-\frac{1}{2} A\right)+\bar{\partial}_{i}\left(U^{i}-\frac{1}{2} B^{i}\right)\right) .
\end{aligned}
$$

Finally, to cancel all spurious terms of order $\Delta t$, we set

$$
R=\frac{1}{2} A, \quad U^{i}=\frac{1}{2} B^{\mathrm{eq}, i},
$$

where $B^{\mathrm{eq}, i}$ denotes the equilibrium part of $B^{i}$ given by

$$
B^{\mathrm{eq}, i}=-\Gamma_{j k}^{i} \Pi^{\mathrm{eq}, j k}-\Gamma_{j k}^{j} \Pi^{\mathrm{eq}, k i}-\Gamma_{k j}^{j} \Pi^{\mathrm{eq}, k i} .
$$

[Note that for second-order accuracy in $\Delta t$, it is sufficient to cancel the equilibrium part of $\Delta t B^{i}$ in Eq. (A24), since the non-equilibrium part, $\Delta t B^{\text {neq, } i}$, is of order $\mathcal{O}\left(\Delta t^{2}\right)$.] With this choice of $R$ and $U^{i}$, Eq. (A24) becomes

$$
\partial_{t} \hat{\rho}+\bar{\partial}_{i}\left(\hat{\rho} \hat{u}^{i}\right)=A+\mathcal{O}\left(\Delta t^{2}\right),
$$

which, after inserting the explicit expression for $A[\mathrm{Eq}$. (A10)], yields the correct continuity equation at order $\mathcal{O}\left(\Delta t^{2}\right)$ :

$$
\partial_{t} \hat{\rho}+\nabla_{i}\left(\hat{\rho} \hat{u}^{i}\right)=0,
$$

where $\nabla$ denotes the covariant derivative.

\section{Momentum Equation}

Adding $\varepsilon\left[\right.$ Eq. (A18)] and $\varepsilon^{2}[$ Eq. (A20)] yields the momentum conservation equation:

$$
\begin{aligned}
\partial_{t}\left(\hat{\rho} \hat{u}^{i}\right)+\bar{\partial}_{j} \Pi^{(0), i j}= & \bar{\partial}_{j} \sigma^{i j}+B^{i}+\frac{1}{\tau} U^{i} \\
& -\frac{\Delta t}{2} \partial_{t} B^{i}+\Delta t\left(1-\frac{1}{2 \tau}\right) \partial_{t} U^{i}
\end{aligned}
$$

Applying the same rescaling of the forcing term as before, $\left(A, B^{i}\right) \rightarrow\left(1-\frac{1}{2 \tau}\right)\left(A, B^{i}\right)$, together with the constraints $\left(R, U^{i}\right)=\frac{1}{2}\left(A, B^{i}\right)$, the momentum equation simplifies to

$$
\partial_{t}\left(\hat{\rho} \hat{u}^{i}\right)+\bar{\partial}_{j} \Pi^{(0), i j}=\bar{\partial}_{j} \sigma^{i j}+B^{i} .
$$

Inserting the explicit expression for $B^{i}$ [Eq. (A11)] and $\Pi^{(0), i j}=\Pi^{\mathrm{eq}, i j}[\mathrm{Eq}$. (A8)] yields the familiar NavierStokes equation:

$$
\partial_{t}\left(\hat{\rho} \hat{u}^{i}\right)+\nabla_{j}\left(\hat{\rho} \hat{u}^{i} \hat{u}^{j}+\hat{\rho} c_{s}^{2} g^{i j}\right)=\nabla_{j} \sigma^{i j},
$$

where $\sigma^{i j}$ denotes the viscous stress tensor, whose explicit form in terms of $\hat{\rho}$ and $\hat{u}^{i}$ can be derived as follows:

$$
\begin{aligned}
\sigma^{i j} \stackrel{(A 21)}{=}-\left(1-\frac{1}{2 \tau}\right) \varepsilon \bar{\sum}_{\lambda} c_{\lambda}^{i} c_{\lambda}^{j} f_{\lambda}^{(1)} \\
\stackrel{(A 14)}{=}\left(\tau-\frac{1}{2}\right) \Delta t \varepsilon \bar{\sum}_{\lambda}\left(c_{\lambda}^{i} c_{\lambda}^{j} D_{t}^{(1)} f_{\lambda}^{(0)}-\mathcal{F}_{\lambda}^{(1)}\right) \\
\quad \approx\left(\tau-\frac{1}{2}\right) \Delta t\left(\bar{\partial}_{k} \Sigma^{\mathrm{eq}, i j k}-C^{i j}\right),
\end{aligned}
$$

where we have assumed that $\left(\tau-\frac{1}{2}\right) \Delta t \partial_{t}^{(1)} \Pi^{(0), i j} \ll 1$. After inserting the explicit expressions for $\Sigma^{\mathrm{eq}, i j k}[\mathrm{Eq}$. (A9)] and $C^{i j}$ [Eq. (A12)], we obtain

$$
\sigma^{i j}=\nu\left(\nabla^{j}\left(\hat{\rho} \hat{u}^{i}\right)+\nabla^{i}\left(\hat{\rho} \hat{u}^{j}\right)+g^{i j} \nabla_{k}\left(\hat{\rho} \hat{u}^{k}\right)\right),
$$

where we have defined $\nu:=\left(\tau-\frac{1}{2}\right) \Delta t c_{s}^{2}$ and neglected terms of the order $\mathcal{O}\left(\hat{u}^{3}\right) \sim \mathcal{O}\left(\mathrm{Ma}^{3}\right)$, Ma being the Mach number.

\section{Newton's algorithm}

The corrected density $\hat{\rho}$ and velocity $\hat{u}^{i}$ are determined implicitly by the coupled Eqs. (A1) and (A2):

$$
\begin{gathered}
\hat{\rho}=\rho+\Delta t R(\hat{\rho}, \hat{u}), \\
\hat{\rho} \hat{u}^{i}=\rho u^{i}+\Delta t U^{i}(\hat{\rho}, \hat{u}),
\end{gathered}
$$

where $R$ and $U^{i}$ are given by Eqs. (7) and (8). Since $R(\hat{\rho}, \hat{u})$ and $U(\hat{\rho}, \hat{u})$ depend only linearly on $\hat{\rho}$, we can eliminate the density by defining $\mathcal{R}(\hat{u}):=R(\hat{\rho}, \hat{u}) / \hat{\rho}$ and $\mathcal{U}(\hat{u}):=U(\hat{\rho}, \hat{u}) / \hat{\rho}$. Now, we can rewrite Eqs. (A1) and (A2) as follows:

$$
\begin{aligned}
\frac{\rho}{\hat{\rho}} & =1-\Delta t \mathcal{R}(\hat{u}), \\
\hat{u}^{i} & =\frac{\rho}{\hat{\rho}} u^{i}+\Delta t \mathcal{U}^{i}(\hat{u}) .
\end{aligned}
$$


Inserting the first equation into the second, we obtain an equation for $\hat{u}$ which is decoupled from the density $\hat{\rho}$ :

$$
\hat{u}^{i}=(1-\Delta t \mathcal{R}(\hat{u})) u^{i}+\Delta t \mathcal{U}^{i}(\hat{u}) .
$$

After defining

$$
G^{i}(\hat{u}):=(1-\Delta t \mathcal{R}(\hat{u})) u^{i}+\Delta t \mathcal{U}^{i}(\hat{u})-\hat{u}^{i},
$$

Newton's algorithm can be used to solve $G^{i}(\hat{u})=0$. To this end, one assigns initial conditions $\hat{u}_{(0)}^{i}=u^{i}$ and iterates,

$$
\hat{u}_{(n+1)}^{i}=\hat{u}_{(n)}^{i}-\left[J^{-1}\right]_{j}^{i} G^{j}\left(\hat{u}_{(n)}\right)
$$

where $J=\left(\frac{d G^{i}}{d \hat{u}^{j}}\right)$ denotes the Jacobian matrix. This procedure converges rapidly, and typically after $1-3$ iterations, a sufficiently accurate solution is found. Given the solution for $\hat{u}$, the solution for $\hat{\rho}$ is obtained from Eq. (A26).

\section{ACKNOWLEDGMENTS}

We acknowledge financial support from the European Research Council (ERC) Advanced Grant No. 319968FlowCCS.
[1] F. Seychelles, Y. Amarouchene, M. Bessafi, and H. Kellay, Phys. Rev. Lett. 100, 144501 (2008).

[2] L. Scriven, Chemical Engineering Science 10, 1 (1959).

[3] K. D. Danov, R. Dimova, and B. Pouligny, Phys. Fluids (1994-present) 12, 2711 (2000).

[4] J. Chomaz, Journal of Fluid Mechanics 442, 387 (2001).

[5] E. R. Priest, Solar Magnetohydrodynamics (Springer, Berlin, 1982).

[6] C. Cao, M. A. Rammaha, and E. S. Titi, Zeitschrift für angewandte Mathematik und Physik ZAMP 50, 341 (1999).

[7] S. Reuther and A. Voigt, Multiscale Model. Simul. 13, 632 (2015).

[8] J. Zhang, S. Childress, A. Libchaber, and M. Shelley, Nature 408, 835 (2000).

[9] M. Arroyo and A. DeSimone, Phys. Rev. E 79, 031915 (2009).

[10] J. W. Barrett, H. Garcke, and R. Nürnberg, Physical Review E 92, 052704 (2015).

[11] D. Hu, P. Zhang, and E. Weinan, Physical Review E 75, 041605 (2007).

[12] J. Fan, T. Han, and M. Haataja, The Journal of chemical physics 133, 235101 (2010).

[13] R. Dimova, S. Aranda, N. Bezlyepkina, V. Nikolov, K. A. Riske, and R. Lipowsky, Journal of Physics: Condensed Matter 18, S1151 (2006).

[14] P. Cicuta, S. L. Keller, and S. L. Veatch, The Journal of Physical Chemistry B 111, 3328 (2007).

[15] M. Ben Amar, J.-M. Allain, N. Puff, and M. I. Angelova, Phys. Rev. Lett. 99, 044503 (2007).

[16] I. Torre, A. Tomadin, A. K. Geim, and M. Polini, Physical Review B 92, 165433 (2015).
[17] M. Mendoza, H. Herrmann, and S. Succi, Phys. Rev. Lett. 106, 156601 (2011).

[18] A. Fasolino, J. Los, and M. I. Katsnelson, Nature materials 6, 858 (2007).

[19] L. D. Landau and E. Lifshitz, The Classical Theory of Fields, Vol. 2 (Butterworth-Heinemann, London, 1975).

[20] J.-D. Debus, M. Mendoza, S. Succi, and H. Herrmann, submitted (2015).

[21] S. Succi, Europhys. Lett. 109, 50001 (2015).

[22] R. Benzi, S. Succi, and M. Vergassola, Physics Reports 222, 145 (1992).

[23] S. Chen and G. D. Doolen, Annual review of fluid mechanics 30, 329 (1998).

[24] M. Mendoza, S. Succi, and H. J. Herrmann, Scientific reports 3, 3106 (2013).

[25] M. Mendoza, J.-D. Debus, S. Succi, and H. J. Herrmann, International Journal of Modern Physics C 25, 1441001 (2014).

[26] J.-D. Debus, M. Mendoza, and H. J. Herrmann, Phys. Rev. E 90, 053308 (2014).

[27] Z. Guo, C. Zheng, and B. Shi, Phys. Rev. E 65, 046308 (2002).

[28] P. L. Bhatnagar, E. P. Gross, and M. Krook, Phys. Rev. 94, 511 (1954).

[29] S. S. Chikatamarla and I. V. Karlin, Phys. Rev. E 79, 046701 (2009).

[30] S. P. Thampi, S. Ansumali, R. Adhikari, and S. Succi, Journal of Computational Physics 234, 1 (2013).

[31] Q. Li, Y. L. He, G. H. Tang, and W. Q. Tao, Phys. Rev. E 81, 056707 (2010). 